Pacific Journal of Mathematics

A PROOF OF THE MOST GENERAL POLYHEDRAL

LesLie C. GLass 


\title{
A PROOF OF THE MOST GENERAL POLYHEDRAL SCHOENFLIES CONJECTURE POSSIBLE
}

\author{
Leslie C. Glaser
}

\begin{abstract}
Here, it is shown that if $M^{n}$ is an $n$-manifold triangulated as a locally finite simplicial complex and $N^{k}$ is a closed subcomplex of int $M^{n}$ that is also a topological $k$-manifold, then $N^{k}$ is topologically locally flat in $M^{n}$ provided $n-k \neq 2$ and each of $N^{k}$ and $M^{n}$ is a simplicial homotopy manifold. This result not only generalizes all known results to date, but also either includes the most general case, where no further assumptions on the triangulations are made, or the general case is false in a very strong sense. That is, if some triangulated topological $n$-manifold is not a simplicial homotopy $n$-manifold, then there exist, for some $m$, a triangulated $m$-sphere $\Sigma$ and $P L(m-1)$-and $(\mathbf{m}+1)$ spheres $S$ and $\mathscr{S}$, respectively, such that $\Sigma$ is a subcomplex of $\mathscr{S}, S$ is a subcomplex of $\Sigma, \mathscr{S}-\Sigma=U \cup V$, where $U$ is homeomorphic to $E^{m+1}$, but $\pi_{1}(V) \neq 0$, and $S$ bounds a $P L \mathrm{~m}$ ball $B$ in $\Sigma$, but $\pi_{1}(\Sigma-B) \neq 0$. The main result is obtained by noting some results related to double suspensions of homotopy 3-and 4-spheres and showing that each open simplex of such a triangulation, as above, is topologically flat in the given manifold.
\end{abstract}

By the Polyhedral Schoenflies Conjecture we will mean the following conjecture:

If the $n$-sphere $S^{n}$ is triangulated as a simplicial complex and $K$ is a subcomplex of $S^{n}$ such that $K$ is topologically homeomorphic to $S^{n-1}$, then $K$ is topologically flat in $S^{n}$ (i.e., $\left(S^{n}, K\right)$ is topologically homeomorphic, as pairs, to $\left(\Sigma \dot{\Delta}^{n}, \dot{\Delta}^{n}\right)$, where $\Sigma$ denotes suspension and $\Delta^{n}$ is the standard $n$-simplex).

More generally, we consider the following conjecture:

If $M^{n}$ is a closed topological $n$-manifold that is triangulated as a simplicial complex, $N^{k}$ is a subcomplex of $M^{n}$ and is also a closed topological $k$-manifold, and $n-k \neq 2$, then $N^{k}$ is topologically locally flat in $M^{n}$.

If the triangulation of $M^{n}$ and the induced triangulation of $N^{k}$ are both $P L$ triangulations, then it is well-known that the conclusion holds ([1] and [17]). In fact, in this case, if $n-k \geqq 3$, then $N^{k}$ is $P L$ locally flat in $M^{n}$ [17]. If $k=n-1$ and both $N^{n-1}$ and $M^{n}$ are star manifolds (an $n$-star manifold is a triangulated manifold such that the link of each $(n-k-1)$-simplex is a $k$-star manifold topologically homeomorphic to $S^{k}$ ), then the conclusion follows by [1]. If $n-k \geqq 3$ 
and both $N^{k}$ and $M^{n}$ are star manifolds, the result follows from [15]. If $k=n-1, M^{n}$ is an $n$-star manifold and $N^{n-1}$ is an $(n-1)$-dimensional $L$-complex (an $L$-complex is a complex $K$ such that, for each simplex $\sigma \in K, \Sigma(l k(\sigma, K))$ is homeomorphic to a topological sphere of dimension $=\operatorname{dim} K-\operatorname{dim} \sigma$ ), the conclusion follows from [12]. It also follows by [1] and [15], that if the second conjecture is valid, then the Polyhedral Schoenflies Conjecture holds, and if $S^{k} \approx N^{k} \subset M^{n} \approx S^{n}(\approx$ denotes topologically homeomorphic), then $\left(M^{n}, N^{k}\right) \approx\left(\Sigma^{n-k} \dot{\Delta}^{k+1}, \dot{\Delta}^{k+1}\right)$.

By a simplicial homotopy n-manifold $M^{n}$ we will mean a locally finite simplicial complex such that the link of each $(n-k-1)$-simplex of $M^{n}$ is a complex homotopy equivalent to $S^{k}$. Here (in Theorems 1 and 3), we show that if $M^{n}$ is a simplicial homotopy $n$-manifold, $N^{k}$ is a simplicial homotopy $k$-manifold that is a subcomplex of $M^{n}$, $n-k \neq 2$, and for $n$ or $k=4$, we also assume that the given complexes are topological manifolds, then $N^{k}$ is topologically locally flat in $M^{n}$. Thus, if $S^{k} \approx N^{k} \subset M^{n} \approx S^{n}, n-k \neq 2$, and each of $N^{k}$ and $M^{n}$ is a simplicial homotopy manifold, then $\left(M^{n}, N^{k}\right) \approx\left(\sum^{n-k} \dot{\Delta}^{k+1}, \dot{\Delta}^{k+1}\right)$.

In Theorem 4, we show that this is the best result possible in that the above results either include the general case, or the general case is false. That is, we show that if the second conjecture is false for some $N^{k} \subset M^{n}$, then one of $N^{k}$ or $M^{n}$ is a triangulated manifold that is not a simplicial homotopy manifold. Moreover, if there exists any closed triangulated topological $n$-manifold that is not a simplicial homotopy $n$-manifold, then the Polyhedral Schoenflies Conjecture is false (for some $m$ ) (refer to Theorem 4). In fact, there would exist counterexamples to the polyhedral Schoenflies problems of both type I and type II considered in [6].

It follows by [14], that if $n \neq 4$, then any simplicial homotopy $n$-manifold is actually a topological $n$-manifold (we include a proof of this fact here in Remark 2). In Theorem 2, we show that if $M^{n}$ is a simplicial homotopy $n$-manifold $(n \geqq 5)$ or is a triangulated closed topological $n$-manifold $(n \leqq 4)$, then each open simplex of $M^{n}$ is topologically locally flat in $M^{n}$. We also should note that if $M^{n}$ is a simplicial homotopy $n$-manifold and $\sigma^{n-k-1} \in M^{n}$, then $L^{k}=l k\left(\sigma^{n-k-1}\right.$, $\left.M^{n}\right)$ is a $P L k$-sphere for $k \leqq 2$, is a $P L$ homotopy 3 -sphere for $k=3$, is a simplicial homotopy 4-manifold homotopy equivalent to $S^{4}$ for $k=4$, and is a simplicial homotopy $k$-manifold topologically homeomorphic to $S^{k}$ for $k \geqq 5$. Furthermore, $\Sigma L^{4} \approx S^{5}$ (refer to the proof of Corollary 2) and $\Sigma^{2} L^{3} \approx S^{5}$ (refer to Remark 1). (Also, see [8] or [14].) However, it is not known whether $\Sigma L^{3}$ is homeomorphic to $S^{4}$, and it is not known whether $L^{4}$ is a topological 4-manifold. In any event, a simplicial homotopy $n$-manifold is more general than the $n$ star manifolds of [1], or the $n$-dimensional $L$-complexes of [12]; and 
they may be more restrictive than the concept of a triangulated topological $n$-manifold. (We note, if $M^{n}$ is a triangulated topological $n$-manifold and $L^{k}=l k\left(\sigma^{n-k-1}, M^{n}\right)$, where $\sigma^{n-k-1} \in M^{n}$, then $\Sigma^{n-k} L^{k} \approx S^{n}$ (refer to [12] or [8]) and $L^{k}$ is homologically equivalent to $S^{k}$; thus a triangulated topological manifold can be thought of as a simplicial homology manifold.)

The concept of simplicial homotopy $n$-manifold extends to complexes having a boundary in the obvious way. In [4], it is shown that if (1) $M_{1}$ and $M_{2}$ are connected simplicial homotopy $n$-manifolds, where $n \geqq 6$ (and both may have a boundary) or where $n=5$ and $B d M_{1}=B d M_{2}=\phi$, (2) $f:\left(M_{1}, B d M_{1}\right) \rightarrow\left(M_{2}, B d M_{2}\right)$ is a $P L$ mapping such that all point-inverses of $f$ and of $\left(f \mid B d M_{1}\right)$ are compact and contractible, and (3) $\varepsilon: M_{1} \rightarrow(0, \infty)$ is a continuous function, then there is a topological homeomorphism $h: M_{1} \rightarrow M_{2}$ such that $d(h(x)$, $f(x))<\varepsilon(x)$ for all $x \in M_{1}$.

In [8], the significance of the following seemingly restrictive question is noted:

Simplified Double Suspension Problem. Does there exist, for some integer $n \geqq 4$, a finite contractible subcomplex $K$ of a $P L$ triangulation of $E^{n}$ such that $\pi_{1}\left(E^{n}-K\right) \neq 0$, but $\left(E^{n} / K\right) \times E^{1}$ is locally Euclidean?

In particular, the equivalence of the following three statements are proven in [8]:

(i) The answer to the Simplified Double Suspension Problem is always NO;

(ii) For all $n \geqq 4$ and all $k \geqq 0$, no $(k+1)$-suspension of any nonsimply connected $P L$ homology $(n-1)$-sphere (i.e., a closed $P L$ $(n-1)$-manifold having the homology groups of $\left.S^{n-1}\right)$ is a manifold ( $\equiv$ is homeomorphic to $S^{n+k}$ ); and

(iii) every triangulated topological $n$-manifold is a simplicial homotopy $n$-manifold.

In fact, if it can be shown that every closed triangulated topological manifold is locally flat on the interior of each 1-simplex, the (i ), (ii) and (iii) above hold, and every closed triangulated topological manifold is locally flat on each open simplex of the triangulation.

We now give some additional definitions and notation. We will use $\cong$ to denote $P L$ homeomorphic (recall $\approx$ denotes topological equivalence). If $X$ is a compact space, $\Sigma X$ denotes the suspension of $X$ (i.e., $X \times[-1,1] / X \times\{-1\}, X \times\{1\}) . \quad \Sigma^{2} X \equiv \Sigma(\Sigma X)$. If $X$ is not compact, we will only consider the case where $X \subset Y, Y$ compact, and 
$\Sigma X$ will be the natural subsuspension of $\Sigma Y . \mathscr{C} X$ will denote the cone over $X$ (i.e., $X \times[0,1] / X \times\{1\}$ ), and $O \mathscr{C} X$ will denote the open cone over $X$ (i.e., $O \mathscr{C} X=(\mathscr{C} X)-X)$.

$E^{n}$ will denote Euclidean $n$-space, $S^{n}$ will denote a space homeomorphic to the unit $n$-sphere in $E^{n+1}$, and $B^{n}$ will denote a space homeomorphic to the unit $n$-ball in $E^{n}$. $\Delta^{n}$ will denote the standard $n$-simplex and $\dot{J}^{n}$ the boundary of $\Delta^{n} . T^{2}$ will denote the 2-torus $S^{1} \times S^{1}$. By a triangulation of a topological manifold $M$, we will mean a pair $(K, h)$, where $K$ is a locally finite simplicial complex and $h$ is a homeomorphism carrying $|K|$ onto $M$. However, we will always suppose that the pair $(K, h)$ is already given, and will consider $h(|K|)=M$ as both a topological manifold and as a complex, without ever mentioning $K$ or $h$. For example, we call the complex $S^{n}\left(\right.$ or $\left.B^{n}\right)$ a $P L n$-sphere ( $n$-ball), if some subdivision of $S^{n}\left(B^{n}\right)$ is simplicially isomorphic to a subdivision of $j^{n+1}\left(\Delta^{n}\right)$ (i.e., if $S^{n} \cong j^{n+1}$ and $B^{n} \cong \Delta^{n}$ ). A manifold $M$ is a $P L n$-manifold, or is a $P L$ triangulated $n$-manifold, if for every vertex $v \in M, l k(v, M)$ is a $P L(n-1)$ ball, or is a $P L(n-1)$-sphere, depending on whether $v \in B d M$, or not.

We write $(X, Y) \approx(A, B)$, if there exists a homeomorphism $h$ carrying $X$ onto $A$ such that $h$ carries the subset $Y$ of $X$ onto the subset $B$ of $A$. If $N^{k}$ is a $k$-manifold contained in the $n$-manifold $M^{n}$, we say $N^{k}$ is topologically locally flat in $M^{n}$, if for every point $x \in N^{k}$, there exists a neighborhood $U$ of $x$ in $M^{n}$ such that

$$
\left(U, U \cap N^{k}\right) \approx\left(E^{n}, E^{k}\right)
$$

(we will only consider locally flat embeddings where $B d N^{k}=B d M^{n}=$ $\phi)$. We say $S^{n-1} \subset S^{n}$ is topologically flat if $\left(S^{n}, S^{n-1}\right) \approx\left(\Sigma \dot{\Delta}^{n}, \dot{\Delta}^{n}\right)$. Finally, we denote the join of two complexes $K$ and $L$ by $K^{*} L$.

2. Results related to double suspensions of homotopy 3spheres.

Proposition 1. Suppose $L^{2}$ is a $P L$ 2-sphere contained as a subcomplex of a PL homotopy 3-sphere $L^{3}, S^{2}$ is a PL 2-sphere contained as a subcomplex of a PL 3-sphere $S^{3}$, and $h: L^{2} \rightarrow S^{2}$ is a homeomorphism carrying $L^{2}$ onto $S^{2}$. Then there exists a homeomorphism $H: \Sigma^{2} L^{3} \rightarrow \Sigma^{2} S^{3}$ carrying $\Sigma^{2} L^{3}$ onto $\Sigma^{2} S^{3}$ such that $H$ carries the suspension circle of $\Sigma^{2} L^{3}$ onto the suspension circle of $\Sigma^{2} S^{3}, H_{\mid \Sigma^{2} L^{2}}$ is a homeomorphism carrying $\Sigma^{2} L^{2}$ onto $\Sigma^{2} S^{2}$, and $H_{1 L^{2}}=h$. (Also, see Corollary 4.3 of [10].)

Proof. The proof follows immediately from Theorem III. 3.2 of 
[8], and for completeness, we will briefly indicate some of the ideas here.

We express $L^{3}$ as the union of two $P L$ homotopy cells $F_{1}^{3}$ and $F_{2}^{3}$, where $L^{3}=F_{1}^{3} \cup F_{2}^{3}$ and $F_{1}^{3} \cap F_{2}^{3}=L^{2}$. Also, we let $S^{3}=B_{1}^{3} \cup B_{2}^{3}$, where $B_{1}^{3} \cap B_{2}^{3}=S^{2}$. It follows from the proof of Theorem III. 3.1 of [8] that the homeomorphism $h \times \mathrm{id}_{T^{2}}: L^{2} \times T^{2} \rightarrow S^{2} \times T^{2}$ extends to homeomorphism $\widetilde{H}_{i}: F_{i}^{3} \times T^{2} \rightarrow B_{i}^{3} \times T^{2}(i=1$ and 2$)$.

In Theorem III. 3.2 (conclusion (i)) of [8], we note that these homeomorphisms induce homeomorphisms $\hat{H}_{i}: F_{i}^{3} \times E^{2} \rightarrow B_{i}^{3} \times E^{2}(i=1$ and 2) such that $\hat{H}_{i \mid L^{2} \times E^{2}}=h \times \mathrm{id}_{E^{2}}$ and each $\hat{H}_{i}$ is bounded on the $E^{2}$ factor. These, in turn (Theorem III. 3.2 (conclusion (ii)) of [8]), induce natural homeomorphisms $H_{i}: \Sigma^{2} F_{i}^{3} \rightarrow \Sigma^{2} B_{i}^{3}(i=1$ and 2$)$ such that $H_{i}$ carries the suspension circle of $\Sigma^{2} F_{i}^{3}$ to the suspension circle of $\Sigma^{2} B_{i}^{3}$ and $H_{1 \mid \Sigma^{2} L^{2}}=H_{2 \mid \Sigma^{2} L^{2}}$ is the natural suspension homeomorphism extending $h$ carrying $\Sigma^{2} L^{2}$ onto $\Sigma^{2} S^{2}$.

We now define $H: \Sigma^{2} L^{3} \rightarrow \Sigma^{2} S^{3}$ by $H_{\mid \Sigma^{2} F_{i}^{3}}=H_{i}(i=1$ and 2$)$.

REMARK 1. It follows from the above that if $F^{3}$ is a homotopy 3-cell and $B^{3}$ is a $P L$ 3-cell such that $B d B^{3}=B d F^{3}=S^{2}$, then $\Sigma^{2} F^{3} \approx \Sigma^{2} B^{3}$ by a homeomorphism that is the identity on $\Sigma^{2} S^{3}$. (This fact is also noted in [14] and a complete proof is given in [8].) Moreover, it follows that $\Sigma^{2} H^{3}=a *\left(b * H^{3} * c\right) * d \approx a *\left(b * S^{3} * c\right) * d=$ $\Sigma^{2} S^{3}$, so that $a * b * c * d$ is carried to itself by the identity map. Hence,

$\left(O \mathscr{C} H^{3}\right) \times E^{1} \approx \Sigma^{2} H^{3}-\{a * c * d\} \approx \Sigma^{2} S^{3}-\{a * c * d\} \approx\left(O \mathscr{C} S^{3}\right) \times E^{1}$

by homeomorphisms, so that $b \times E^{1}$ is carried to itself by the identity map. Thus, if $L^{4}$ is a simplicial homotopy 4-manifold, then $L^{4} \times E^{1}$ is a 5-manifold. That is, if $v$ is a vertex of $L^{4}$, then

$$
\text { st }\left(v, L^{4}\right) \times E^{1} \approx\left\{O \mathscr{C}\left(l k\left(v, L^{4}\right)\right)\right\} \times E^{1} \approx E^{5}
$$

(since $l k\left(v, L^{4}\right)$ is a $P L$ homotopy 3 -sphere). (Also see [14].)

Corollary 1. If $L^{3}$ is a PL homotopy 3-sphere contained as a subcomplex of a simplicial homotopy 4-manifold $L^{4}$, then $L^{3} \times E^{1}$ is locally flat in $L^{4} \times E^{1}$ (recall $L^{4} \times E^{1}$ is a topological 5-manifold). Moreover, if $L^{3} \subset L^{4}$ is as above, and $O \mathscr{C} L^{3} \approx E^{4}$ and $L^{4}$ is homotopy equivalent to $S^{4}$, then $\left(O C \mathscr{C} L^{4}, O \mathscr{C} L^{3}\right)$ is homeomorphic as pairs to $\left(E^{5}, E^{4}\right)$, where $O \mathscr{C} L^{3} \subset O \mathscr{C} L^{4}$ is the natural embedding as an open subcone and $E^{4}=E^{4} \times 0 \subset E^{5}$.

Proof. Let $\Sigma L^{4}=v^{*} L^{4} * w$ be the suspension of $L^{4}$ from the vertices $v$ and $w$. Then $\Sigma L^{3}=v * L^{3} * w$ is a subcomplex of $\Sigma L^{4}$. 
Clearly, $\left(\Sigma L^{4}-\{v \cup w\}, \Sigma L^{3}-\{v \cup w\}\right)$ is homeomorphic as pairs to $\left(L^{4} \times E^{1}, L^{3} \times E^{1}\right)$. Let $f$ denote the natural homeomorphism carrying the former pair onto the latter pair.

Let $x$ denote an arbitrary vertex of $L^{3} \subset L^{3}$. Then $l k\left(x, L^{3}\right)=\widetilde{L}^{2}$ is a $P L 2$-sphere and $l k\left(x, L^{4}\right)=\widetilde{L}^{3}$ is a $P L$ homotopy 3 -sphere. We now think of the pair $\left(\widetilde{L}^{3}, \widetilde{L}^{2}\right)$ as the pair $\left(L^{3}, L^{2}\right)$ of Proposition 1. For the pair $\left(S^{3}, S^{2}\right)$ of Proposition 1, we use the standard $(3,2)$-sphere pair (i.e., $\left.\left(S^{3}, S^{2}\right)=\left(\Sigma \dot{\Delta}^{3}, \dot{\Delta}^{3}\right)\right)$. We now claim that the fact that $L^{3} \times E^{1}$ is locally flat in $L^{4} \times E^{1}$ follows immediately from Proposition 1.

That is, by Proposition 1, there exists a homeomorphism

$$
H:\left(\Sigma^{2} \widetilde{L}^{3}, \Sigma^{2} \widetilde{L}^{2}, \widetilde{L}^{2}\right) \longrightarrow\left(\Sigma^{2} S^{3}, \Sigma^{2} S^{2}, S^{2}\right),
$$

carrying the first suspension circle to the second suspension circle in a natural way. Hence, thinking of $\Sigma^{2} \widetilde{L}^{3}$ as $v *\left(x * \widetilde{L}^{3} * y\right) * w$ and $\Sigma^{2} S^{3}$ as $a *\left(b * S^{3} * c\right) * d, H$ can be defined so as to carry $v * x * y * w$ linearly onto the suspension circle $a * b * c * d$, with $(v, x, y, w)$ going to $(a, b$, $c, d)$. Now $\left[\left(v *\left(x * \widetilde{L}^{3} * y\right) * w\right)-(v * y * w)\right]$ is homeomorphic to

$$
\left(v *\left[\left(x * \widetilde{L}^{3}\right)-\widetilde{L^{3}}\right] * w\right)-\{v \cup w\} \subset v *\left(x * \tilde{L}^{3}\right) * w \subset v * L^{4} * w=\Sigma L^{4}
$$

in a natural manner. Let us denote the homeomorphism going from the former expression to the first given subset of $\Sigma L^{4}$ by $g$. Let $k=H^{-1} \mid\left(\Sigma^{2} S^{3}\right)-(a * * * d)$.

Then the composition

$$
f \circ g \circ k:\left(\left(\Sigma^{2} S^{3}\right)-(a * c * d),\left(\Sigma^{2} S^{2}\right)-(a * c * d)\right) \longrightarrow\left(L^{4} \times E^{1}, L^{3} \times E^{1}\right)
$$

shows that $f\left(\left(v *\left[\left(x * \widetilde{L}^{2}\right)-\widetilde{L}^{2}\right] * w\right)-\{v \cup w\}\right) \subset L^{3} \times E^{1}$ is locally flat in $L^{4} \times E^{1}$. Since, as $x$ varies over the vertices of $L^{3}$, these open subsets of $L^{3} \times E^{1}$ form an open cover of $L^{3} \times E^{1}$, it follows that $L^{3} \times E^{1}$ is locally flat in $L^{4} \times E^{1}$.

We now want to show that $\left(O \mathscr{C} L^{4}, O \mathscr{C} L^{3}\right) \approx\left(E^{5}, E^{4}\right)$, if $O \mathscr{C} L^{3} \approx E^{4}$ and $L^{4}$ is homotopy equivalent to $S^{4}$. Since $L^{4} \times E^{1}$ is a 5-manifold, it follows from [12] (or [8], Theorem III. 3.3, (3)), that $\Sigma L^{4} \approx E^{5}$ and hence $O \mathscr{C} L^{4} \approx E^{5}$. If $v$ denotes the vertex of $O \mathscr{C} L^{4}$, since

$$
\left(O \mathscr{C} L^{4}-\{v\}, O \mathscr{C} L^{3}-\{v\}\right) \approx\left(L^{4} \times E^{1}, L^{3} \times E^{1}\right),
$$

it follows from the above, that $O \mathscr{C} L^{3} \subset O \mathscr{C} L^{4}$ is closed embedding of $E^{4}$ in $E^{5}$ that is locally flat modulo the point $v \in O \mathscr{C} L^{3}$. By [11], it follows that $O \mathscr{C} L^{3}$ is flat in $O \mathscr{C} L^{4}$. Hence,

$$
\left(O \mathscr{C} L^{4}, O \mathscr{C} L^{3}\right) \approx\left(E^{5}, E^{4}\right) .
$$

COROLlary 2. Suppose $N^{4}$ is a simplicial homotopy 4-manifold 
contained as a subcomplex of the simplicial homotopy 5-manifold $M^{5}$. If $N^{4}$ is a topological 4-manifold, then $N^{4}$ is topologically locally flat in $M^{5}$.

Proof. Let $v$ denote an arbitrary vertex of $N^{4}$. We denote $l k\left(v, N^{4}\right)$ by $L^{3}$ and $l k\left(v, M^{3}\right)$ by $L^{4}$. Then $s t\left(v, N^{4}\right)=v * L^{3}$ and $s t\left(v, M^{5}\right)=v * L^{4}$. Since $N^{4}$ is a topological 4-manifold, $O \mathscr{C} L^{3} \approx E^{4}$. By [14], $M^{5}$ is a topological 5-manifold. (Actually, this is easy to see as follows: Since $L^{4}$ is the link of a vertex of $M^{5}$, it is a simplicial homotopy 4-manifold that is homotopy equivalent to $S^{4}$. By Remark $1, L^{4} \times E^{1}$ is a 5 -manifold and by Corollary $1, O \mathscr{C} L^{4} \approx E^{5}$. Thus $s t\left(v, M^{5}\right) \approx E^{5}$, for each vertex $v$ in $M^{5}$, and $M^{5}$ is a topological 5-manifold.)

Thus, by Corollary $1,\left(O \mathscr{C} L^{4}, O \mathscr{C} L^{3}\right) \approx\left(E^{5}, E^{4}\right)$. Since $v$ was an arbitrary vertex of $N^{4}$ and the open stars of vertices of $N^{4}$ form an open cover of $N^{4}$, it follows that $N^{4}$ is locally flat in $M^{5}$.

Remark 2. It is now easy to see that any simplicial homotopy $n$-manifold $M^{n}$ is a topological $n$-manifold, provided $n \neq 4$. That is, for $n \leqq 3$, it is trivial to see that simplicial homotopy $n$-manifolds are $P L n$-manifolds. For $n=5$, the result follows from the comments above. For $n \geqq 6$, the result follows by induction, since $l k\left(v, M^{n}\right)=$ $L^{n-1}$ is a simplicial homotopy $(n-1)$-manifold that is homotopy equivalent to $S^{n-1}$. Since $n-1 \geqq 5, L^{n-1}$ is a topological $(n-1)$-manifold by induction, and by [5], $L^{n-1} \approx S^{n-1}$. We recall, the problem when $n=4$, is that it is unknown whether the suspension of an arbitrary homotopy 3 -sphere is topologically $S^{4}$ or not.

3. Crossing with $T^{2}$ and results related to simplicial Homotopy 4-manifolds.

Proposition 2. Suppose $K$ is a contractible finite homogeneous 4-complex (i.e., every simplex of $K$ lies in some 4-dimensional simplex of $K)$ such that the boundary of $K(\bmod 2)$, say $L^{3}$, is a $P L$ homotopy 3-sphere and $K \times E^{2}$ is a 6-manifold with boundary $L^{3} \times E^{2}$. Then there exists a homeomorphism $h: L^{3} \times T^{2} \rightarrow S^{3} \times T^{2}$ and a homeomorphism $H: K \times T^{2} \rightarrow B^{4} \times T^{2}\left(B d B^{4}=S^{3}\right)$ extending $h$ such that

$$
H_{*}: \pi_{1}\left(K \times T^{2}\right) \longrightarrow \pi_{1}\left(B^{4} \times T^{2}\right)
$$

commmutes with the projection to $\pi_{1}\left(T^{2}\right)$.

Proof. Let $B^{3}$ denote a $P L 3$-ball in $L^{3}$ and let $F^{3}=L^{3}$-int $B^{3}$. Then $F^{3}$ is a $P L$ homotopy 3-cell with $B d F^{3}=B d B^{3}$. Let 
$i: B d F^{3} \rightarrow B d B^{3}$ denote the identity homeomorphism. By Theorem III. 3.1 of [8], the identity homeomorphism

$$
i \times \text { id. }_{T^{2}}: B d F^{3} \times T^{2} \longrightarrow B d B^{3} \times T^{2}
$$

extends to a homeomorphism $\tilde{h}: F^{3} \times T^{2} \rightarrow B^{3} \times T^{2}$. In fact, if $j_{1}: B d F^{3} \times[0,1) \rightarrow F^{3}$ defines a collar of $B d F^{3}$ in $F^{3}$ and, thinking of $B^{3}$ as a cone $v * B d B^{3}, j_{2}: B d B^{3} \times[0,1) \rightarrow\left(v * B d B^{3}\right)-\{v\} \subset B^{3}$ is the natural homeomorphism, then the homeomorphism

$$
\tilde{h}: F^{3} \times T^{2} \longrightarrow B^{3} \times T^{2}
$$

can be chosen so that for some $t, 0<t<1, \tilde{h}_{\mid j_{1}\left(B d F^{3} \times[0, t]\right) \times T^{2}}$ is the following composition of homeomorphisms

$$
\begin{gathered}
j_{1}\left(B d F^{3} \times[0, t]\right) \times T^{2} \stackrel{j_{1}^{-1} \times \text { id. } T^{2}}{\longrightarrow}\left(B d F^{3} \times[0, t]\right) \times T^{2} \stackrel{i \times \text { id. }[0, t] \times \text { id. } T^{2}}{\longrightarrow} \\
\left(B d B^{3} \times[0, t]\right) \times T^{2} \stackrel{j_{2} \times \text { id. } T^{2}}{\longrightarrow} j_{2}\left(B d B^{3} \times[0, t]\right) \times T^{2} \subset B^{3} \times T^{2} .
\end{gathered}
$$

Clearly $\tilde{h}_{*}: \pi_{1}\left(F^{3} \times T^{2}\right) \rightarrow \pi_{1}\left(B^{3} \times T^{2}\right)$ commutes with the projection to $\pi_{1}\left(T^{2}\right)$.

Let $j_{3}:[t / 2, t] \rightarrow[t / 2,1]$ be the linear homeomorphism sending $t / 2$ to $t / 2$ and $t$ to 1 . Let $\hat{k}: F^{3} \rightarrow B^{3}$ denote the homotopy equivalence defined by

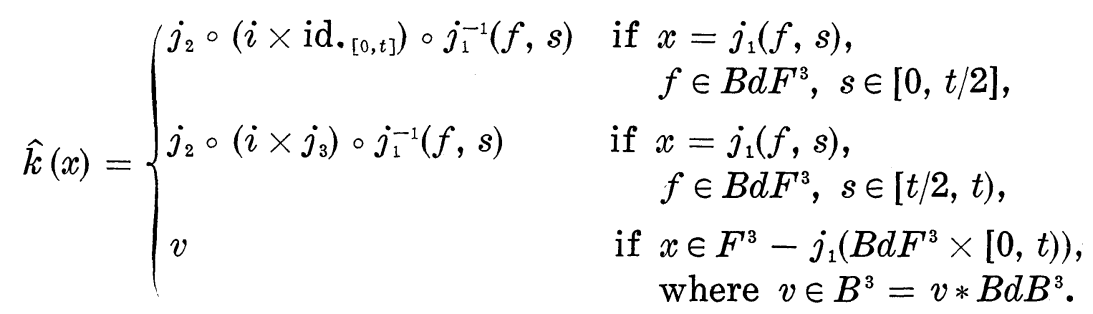

Let $\tilde{k}: F^{3} \times T^{2} \rightarrow B^{3} \times T^{2}$ be the homotopy equivalence defined by $\widetilde{k}=\hat{k} \times$ id. $T_{T^{2}}$. We note that $\widetilde{k}=\widetilde{h}$ on $j_{1}\left(B d F^{3} \times[0, t / 2]\right) \times T^{2}=Z$. Also, it is not too difficult to see that $\widetilde{h}$ is homotopic $\bmod Z$ to $\widetilde{k}$. (That is, $\tilde{h}$ is clearly homotopic $\bmod Z$ to a map $\widetilde{k}^{\prime}: F^{3} \times T^{2} \rightarrow B^{3} \times T^{2}$ such that $\widetilde{k}^{\prime}=\widetilde{k}$ on $j_{1}\left(B d F^{3} \times[0, t]\right) \times T^{2}$ and

$$
\tilde{k}^{\prime}\left(\left(F^{3} \times T^{2}\right)-\left\{j_{1}\left(B d F^{3} \times[0, t)\right) \times T^{2}\right\} \subset\{v\} \times T^{2} .\right.
$$

By taking a large enough $2^{m}$-fold covering of each of $F^{3} \times T^{2}$ and $B^{3} \times T^{2}$, if necessary, we can suppose we have covering maps, which we still call $\widetilde{h}, \widetilde{k}$, and $\widetilde{k}^{\prime}$ with properties as above, so that projection on the $T^{2}$ factor moves points less than any pre-assigned small number. But then, $\widetilde{k}^{\prime}$ will clearly be homotopic to $\tilde{k}$

$$
\bmod \left\{j_{1}\left(B d F^{3} \times[0, t]\right) \times T^{2}\right\} .
$$


Let $\widetilde{G}: F^{3} \times T^{2} \times[0,1] \rightarrow B^{3} \times T^{2}$ denote a homotopy $\bmod Z$ between $\tilde{h}$ and $\widetilde{k}$, where $\widetilde{G}_{0}=\widetilde{h}$ and $\widetilde{G}_{1}=\widetilde{k}$. Clearly, $\widetilde{h}$ extends to a homeomorphism $h: L^{3} \times T^{2} \rightarrow S^{3} \times T^{2}$. That is, thinking of $S^{3}$ as $2 B^{3}$, the double of $B^{3}, \widetilde{h}$ extends by the identity homeomorphism (recall $L^{3}=F^{3} \cup B^{3}$ ). Similarly, $\widetilde{k}$ extends to a homotopy equivalence $k: L^{3} \times T^{2} \rightarrow S^{3} \times T^{2}$ such that $k=h$ on $Z \cup\left(B^{3} \times T^{2}\right)$. Also, $\widetilde{G}$ extends to a homotopy $G: L^{3} \times T^{2} \times[0,1] \rightarrow S^{3} \times T^{2}$ such that $G_{0}=h$ and $G_{1}=k$.

We now suppose that $B^{4}$ is a 4 -ball such that $B d B^{4}=S^{3}$. Since $K$ is contractible and $B^{4}$ is contractible, the map

$$
k \cup \text { id. }{ }_{B^{3}}: L^{3}\left(=F^{3} \cup B^{3}\right) \rightarrow S^{3}\left(=B^{3} \cup B^{3}\right)
$$

extends to a homotopy equivalence $\tilde{g}: K \rightarrow B^{4}$. Let $g: K \times T^{2} \rightarrow B^{4} \times T^{2}$ be the homotopy equivalence defined by $g=\widetilde{g} \times \mathrm{id}$. $\tau^{2}$. We note $g=k$ on $B d\left(K \times T^{2}\right)=L^{3} \times T^{2}$. Let $j_{3}: L^{3} \times T^{2} \times[0,3] \rightarrow K \times T^{2}$ be a collar of $L^{3} \times T^{2}$ in $K \times T^{2}$ [1] (since $K \times E^{2}$ is a 6-manifold with boundary $L^{3} \times E^{2}, K \times T^{2}$ is a 6-manifold with boundary $\left.L^{3} \times T^{2}\right)$. By making use of the maps $h \times \mathrm{id}_{{ }_{[0,1]}}, G$, and $g$ we can define a homotopy equivalence

$$
\tilde{H}: K \times T^{2} \longrightarrow B^{4} \times T^{2},
$$

extending $h: L^{3} \times T^{2} \rightarrow S^{3} \times T^{2}$, such that $\tilde{H} \mid j_{3}\left(L^{3} \times T^{2} \times[0,1]\right)$ is a homeomorphism. That is, we "apply", in the appropriate manner, $h \times \mathrm{id} .\left[{ }_{[n]}\right.$ on $j_{3}\left(L^{3} \times T^{2} \times[0,1]\right), G$ on $j_{3}\left(L^{3} \times T^{2} \times[1,2]\right)$, and $g$ on $\left(K \times T^{2}\right)-j_{3}\left(L^{3} \times T^{2} \times[0,2)\right)$.

$$
\widetilde{H}_{*}: \pi_{1}\left(K \times T^{2}\right) \longrightarrow \pi_{1}\left(B^{4} \times T^{2}\right)
$$

commutes with projection to $\pi_{1}\left(T^{2}\right)$, since $h_{*}: \pi_{1}\left(L^{3} \times T^{2}\right) \rightarrow \pi_{1}\left(S^{3} \times T^{2}\right)$ does.

The claim is now, by applying the techniques of the purely geometrical proof of Theorem III. 3.1 of [8], we can define a homeomorphism $H: K \times T^{2} \rightarrow B^{4} \times T^{2}$ so that for some $t, 0<t<1, H=\widetilde{H}$ on $j_{3}\left(L^{3} \times T^{2} \times[0, t]\right)$. Hence, such a homeomorphism $H$ will extend $h$ and $H_{*}: \pi_{1}\left(K \times T^{2}\right) \rightarrow \pi_{1}\left(B^{4} \times T^{2}\right)$ will commute with the projection to $\pi_{1}\left(T^{2}\right)$. That is, the long, but elementary, 8-step proof given for Theorem III. 3.1 of [8] applies here, essentially word for word, except the homotopy equivalence $g: D \times T^{k} \rightarrow B^{n} \times T^{k}$ used there is now replaced by $H: K \times T^{2} \rightarrow B^{4} \times T^{2}$ (also $A \subset D$ corresponds to $L^{3} \subset K$ ). The proof only requires some of the geometrical results of [5] and [7].

CoRollary 3. Suppose $L^{3}$ is a PL homotopy 3-sphere contained as a subcomplex of a simplicial homotopy 4-manifold $L^{4}$. If $L^{4}$ is homotopy equivalent to $S^{4}$ then

(i ) $\left(L^{4} \times E^{2}, L^{3} \times E^{2}\right) \approx\left(S^{4} \times E^{2}, S^{3} \times E^{2}\right)$, as pairs, so that the 
homeomorphism is bounded on the $E^{2}$ factor (here $\left.\left(S^{4}, S^{3}\right) \approx\left(\Sigma \dot{\Delta}^{4}, \dot{\Delta}^{4}\right)\right)$, and

(ii) $\left(\Sigma^{2} L^{4}, \Sigma^{2} L^{3}\right) \approx\left(\Sigma^{2} S^{4}, \Sigma^{2} S^{3}\right)$, as pairs, so that the homeomorphism carries the first suspension circle canonically onto the second suspension circle (as in Proposition 1). (Also see Corollary 5.4 of [10].)

Proof. Since $L^{3} \subset L^{4}$ and $L^{4}$ is homotopy equivalent to $S^{4}$, we can express $L^{4}$ as $K_{1} \cup K_{2}$, where $K_{1} \cap K_{2}=L^{3}$ and each of $K_{1}$ and $K_{2}$ is a contractible finite homogeneous 4-complex. We claim $K_{i} \times E^{2}(i=1$ and 2) is a 6-manifold with boundary $L^{3} \times E^{2}$. For, by Corollary 1, $L^{3} \times E^{1}$ is locally flat in the 5-manifold $L^{4} \times E^{1}$. Hence $L^{3} \times E^{2}$ is locally flat in the 6-manifold $L^{4} \times E^{2}$ and $L^{3} \times E^{2}$ separates $L^{4} \times E^{2}$ into the two 6-manifolds $K_{1} \times E^{2}$ and $K_{2} \times E^{2}$.

Hence, we now can apply Proposition 2 to each of $K_{1}$ and $K_{2}$ using the same $h: L^{3} \times T^{2} \rightarrow S^{3} \times T^{2}$ in each case. That is, for $i=1$ and 2, there exists a homeomorphism $H_{i}: K_{i} \times T^{2} \rightarrow B_{i}^{4} \times T^{2}$ extending $h$ such that $\left(H_{i}\right)_{*}: \pi_{1}\left(K_{i} \times T^{2}\right) \rightarrow \pi_{1}\left(B_{i}^{4} \times T^{2}\right)$ commutes with the projection to $\pi_{1}\left(T^{2}\right)$. Let $H: L^{4} \times T^{2} \rightarrow S^{4} \times T^{2}$ be the homeomorphism defined by $H_{\mid K_{i} \times T^{2}}=H_{i}$, where $S^{4}=B_{1}^{4} \cup B_{2}^{4}$. We note $H_{\mid L^{3} \times T^{2}}$ carries $L^{3} \times T^{2}$ onto $S^{3} \times T^{2}$ and $H_{*}: \pi_{1}\left(L^{4} \times T^{2}\right) \rightarrow \pi_{1}\left(S^{4} \times T^{2}\right)$ commutes with the projection to $\pi_{1}\left(T^{2}\right)$. But then, any homeomorphism $\tilde{H}: L^{4} \times E^{2} \rightarrow S^{4} \times E^{2}$ covering $H$ is bounded on the $E^{2}$ factor. That is, $\widetilde{H}$ satisfies $\left\|y-p_{2} \circ \widetilde{H}(x, y)\right\|<$ constant, for all $(x, y) \in L^{4} \times E^{2}$, where $p_{2}$ denotes the projection $p_{2}: S^{4} \times E^{2} \rightarrow E^{2}$. Thus

$$
\left(L^{4} \times E^{2}, L^{3} \times E^{2}\right) \approx\left(S^{4} \times E^{2}, S^{3} \times E^{2}\right)
$$

so as to be bounded on the $E^{2}$ factor and hence conclusion (i ) holds.

By embedding $L^{4} \times E^{2}$ in $\Sigma^{2} L^{4}=L^{4} * S^{1}$ and $S^{4} \times E^{2}$ in $\Sigma^{2} S^{4}=$ $S^{4} * S^{1}$ in the obvious manner, a homeomorphism $\hat{H}: \Sigma^{2} L^{4} \rightarrow \Sigma^{2} S^{4}$ can be defined simply as the composition

$$
\left(L^{4} * S^{1}\right)-S^{1} \longleftarrow L^{4} \times E^{2} \stackrel{\check{H}}{\longrightarrow} S^{4} \times E^{2} \longrightarrow\left(S^{4} * S^{1}\right)-S^{1}
$$

on $\left(L^{4} * S^{1}\right)-S^{1}$ and the identity: $S^{1} \rightarrow S^{1}$ on $S^{1}$ (refer to the proof of Theorem III. 3.2, (ii) of [8]). Thus $\left(\Sigma^{2} L^{4}, \Sigma^{2} S^{3}\right) \approx\left(\Sigma^{2} S^{4}, \Sigma^{2} S^{3}\right)$ and (ii) holds.

COROLLARY 4. Suppose $L^{4}$ is a simplicial homotopy 4-manifold contained as a subcomplex of a simplicial homotopy 5-manifold $L^{5}$. If $L^{i}$ is homotopy equivalent to $S^{i}$, for $i=4$ and 5 , then

$$
\left(O \mathscr{C} L^{5}, O^{\prime} \mathscr{C} L^{4}\right) \approx\left(E^{6}, E^{5}\right),
$$

where $O \mathscr{C} L^{4} \subset O \mathscr{C} L^{5}$ is the natural embedding as an open subcone and $E^{5}=E^{5} \times 0 \subset E^{6}$. 
Proof. Since $L^{5}$ is a 5-manifold ([14] or Remark 2) homotopy equivalent to $S^{5}, L^{5} \approx S^{5}$ [5]. Thus $O \mathscr{C} L^{5} \approx E^{6}$. As we have noted earlier, since $L^{4}$ is homotopy equivalent to $S^{4}, \Sigma L^{4} \approx S^{5}$ and $O \mathscr{C} L^{4} \approx E^{5}$.

The proof is now quite similar to the proof of Corollary 1. That is, let $\Sigma L^{5}=v * L^{5} * w\left(\approx S^{6}\right)$. Then $\Sigma L^{4}=v * L^{4} * w$ is a subcomplex of $\Sigma L^{5}$. Let $x$ denote an arbitrary vertex of $L^{4}$. Then $l k\left(x, L^{5}\right)=\widetilde{L}^{4}$ is a simplicial homotopy 4-manifold homotopy equivalent to $S^{4}$ and $l k\left(x, L^{4}\right)=\widetilde{L}^{3}$ is a $P L$ homotopy 3 -sphere. By Corollary 3 ,

$$
\left(v *\left(x * \widetilde{L}^{4} * y\right) * w, v *\left(x * \widetilde{L}^{3} * y\right) * w\right) \approx\left(a *\left(b * S^{4} * c\right) * d, a *\left(b * S^{3} * c\right) * d\right),
$$

as pairs, with $v *(x * y) * w$ going to $a *(b * c) * d$. Then, as in the proof of Corollary 1, it follows that $O \mathscr{C} L^{4} \subset O \mathscr{C} L^{5}$ is a closed embedding of $E^{5}$ in $E^{6}$ that is locally flat modulo the vertex of the open cone $O \mathscr{C} L^{4}$. Again by [11], $O \mathscr{C} L^{4}$ is flat in $O \mathscr{C} L^{5}$ and hence

$$
\left(O \mathscr{C} L^{5}, O \mathscr{C} L^{4}\right) \approx\left(E^{6}, E^{5}\right)
$$

Corollary 5. Suppose $N^{5}$ is a simplicial homotopy 5-manifold contained as a subcomplex of the simplicial homotopy 6-manifold $M^{6}$. Then $N^{5}$ is a topological 5-manifold embedded in the topological 6manifold $M^{6}$ so as to be topologically locally flat.

Proof. The proof follows by Remark 2 and by considering link pairs, making use of Corollary 4, as we did in the proof of Corollary 2, where we made use of Corollary 1.

\section{The main results.}

THEOREM 1. Suppose $M^{n}(n \geqq 1)$ is a simplicial homotopy $n$ manifold, $N^{n-1}$ is a simplicial homotopy (n-1)-manifold that is a subcomplex of $M^{n}$, and when $n$ or $n-1=4$ the corresponding complex is also a topological (closed) $n$ or $(n-1)$-manifold. Then $N^{n-1}$ is a topological closed (n-1)-manifold embedded in the topological n-manifold $M^{n}$ so as to be topologically locally flat. Moreover, if $N^{n-1} \approx S^{n-1}$ and $M^{n} \approx S^{n}$, then $\left(M^{n}, N^{n-1}\right) \approx\left(\Sigma \Delta^{\dot{n}}, \Delta^{\dot{n}}\right)$.

Proof. We first recall that $M^{n}$ is a topological $n$-manifold and $N^{n-1}$ is a topological $(n-1)$-manifold for all $n \geqq 1$. This follows by [14], or Remark 2, for $n$ or $n-1 \neq 4$, and by assumption, for $n$ or $n-1=4$.

For $n \leqq 3$, each of $M^{n}$ and $N^{n-1}$ are $P L$ manifolds and the result is well known (in fact, $N^{n-1}$ is $P L$ locally flat in $M^{n}$ for these cases). We now consider $N^{3} \subset M^{4}$. Let $v$ denote an arbitrary vertex of $N^{3}$. Then $\left(l k\left(v, M^{4}\right), l k\left(v, N^{3}\right)\right)=\left(L^{3}, L^{2}\right)$ is a pair, such that $L^{3}$ is a $P L$ 
homotopy 3 -sphere and $L^{2}$ is a $P L 2$-sphere. Thus $L^{2}$ is $(P L)$ locally flat in $L^{3}$. Since $M^{4}$ is a topological 4-manifold, $O \mathscr{C} L^{3} \approx E^{4}$. Hence, since for any vertex $v \in N^{3}$, the corresponding $L^{2} \subset L^{3}$ is locally flat, it follows that given any $v \in N^{3}, O \mathscr{C} L^{2} \subset O \mathscr{C} L^{3}$ is a closed embedding of $E^{3}$ in $E^{4}$ so as to be locally flat except perhaps for the vertex $v$ of the open cone $O \mathscr{C} L^{2}$. By [11], $O \mathscr{C} L^{2}$ is flat in $O \mathscr{C} L^{3}$ and hence, $N^{3}$ is topologically locally flat in $M^{4}$. Hence, for $n \leqq 4$, if $N^{n-1} \approx S^{n-1}$ and $M^{n} \approx S^{n}$, since $N^{n-1}$ is locally flat in $M^{n}$, it follows by [1] that $\left(M^{n}, N^{n-1}\right) \approx\left(\Sigma \dot{\Delta}^{n}, \dot{\Delta}^{n}\right)$.

For $n=5$ or 6 , the result follows by Corollary 2 or Corollary 5, respectively. The proof for $n \geqq 7$ now follows by induction. That is, consider $N^{n-1} \subset M^{n}$ for some fixed $n \geqq 7$ and suppose we know the result for all $k, 1 \leqq k<n$. Let $v$ be a vertex of $N^{n-1}$ and consider the pair $\left(l k\left(v, M^{n}\right), l k\left(v, N^{n-1}\right)\right)=\left(L^{n-1}, L^{n-2}\right)$. Since $n-2 \geqq 5, L^{n-2}$ is a topological $(n-2)$-sphere and $L^{n-1}$ is a topological (n-1)-sphere (refer to Remark 2). By induction, $L^{n-2} \subset L^{n-1}$ is topologically locally flat. By [1], $\left(L^{n-1}, L^{n-2}\right) \approx\left(\Sigma \dot{\Delta}^{n-1}, \dot{\Delta}^{n-1}\right)$. Hence

$$
\left(O \mathscr{C} L^{n-1}, O \mathscr{C} L^{n-2}\right) \approx\left(E^{n}, E^{n-1}\right),
$$

and it follows that $N^{n-1}$ is topologically locally flat in $M^{n}$. Therefore, by induction and [1], the result follows for all $n$.

CoRollary 6. Suppose $M^{n}$ is a topological closed n-manifold triangulated as a simplicial complex and $N^{n-1}$ is a topological closed (n-1)-manifold contained in $M^{n}$ as a subcomplex. If either

(1) $n \leqq 5$, and for $n=5$, we assume that the given triangulation of $M^{5}$ also makes it into a simplicial homotopy 5-manifold, or

(2) the answer to the Simplifield Double Suspension Problem is always NO (refer to the introduction), then $N^{n-1}$ is locally flat in $M^{n}$. Also, if $N^{n-1} \approx S^{n-1}$ and $M^{n} \approx S^{n}$, then $\left(M^{n}, N^{n-1}\right) \approx\left(\Sigma \dot{\Delta}^{n}, \dot{\Delta}^{n}\right)$.

Proof. The result in case (1) follows from Theorem 1, since any triangulated closed topological $n$-manifold is a simplicial homotopy $n$-manifold, if $n \leqq 4$. The result in case (2) also follows from Theorem 1, since, by [8], our assumption implies that all triangulated manifolds are simplicial homotopy manifolds (also recall our comments in the introduction).

THeorem 2. Suppose $M^{n}$ is either (i) a simplicial homotopy $n$ manifold, where $n \geqq 5$, or (ii) a triangulated closed topological $n$ manifold, where $n \leqq 4$. Then $M^{n}$ is triangulated so as to be locally flat on each open simplex. 
Proof. We first note, given any $(n-k-1)$-simplex $\sigma^{n-k-1} \in M^{n}$, then int $\sigma^{n-k-1}$ has a neighborhood $U=s t\left(\sigma^{n-k-1}, M^{n}\right)$ homeomorphic to $\left(O \mathscr{C} L^{k}\right) \times E^{n-k-1}$, where $L^{k}=l k\left(\sigma^{n-k-1}, M^{n}\right)$. In fact, we claim that $\left(U\right.$, int $\left.\sigma^{n-k-1}\right) \approx\left(\left(O \mathscr{C} L^{k}\right) \times E^{n-k-1}, c \times E^{n-k-1}\right)$ as pairs, where $c$ is the "vertex" of $O \mathscr{C} L^{k}$. That is, given any

$$
\sigma^{n-k-1} \in M^{n}, \sigma^{n-k-1} * L^{k}=\operatorname{st}\left(\sigma^{n-k-1}, M^{n}\right)
$$

is a closed neighborhood of int $\sigma^{n-k-1}$. Let $\beta\left(\sigma^{n-k-1}\right)$ denote the barycenter of $\sigma^{n-k-1}$. Then

$$
\sigma^{n-k-1} * L^{k} \cong \dot{\sigma}^{n-k-1} *\left\{\beta\left(\sigma^{n-k-1}\right) * L^{k}\right\} \approx S^{n-k-2} *\left\{c * L^{k}\right\},
$$

with $\sigma^{n-k-1}$ carried to $S^{n-k-2} * c$ in a natural fashion. Now

$$
\left(S^{n-k-2} *\left\{c * L^{k}\right)\right\}-S^{n-k-2} \approx E^{n-k-1} \times\left\{c * L^{k}\right\},
$$

in a natural fashion, with $\left(S^{n-k-2} * c\right)-S^{n-k-2}$ going to $E^{n-k-1} \times c$. Hence, the natural homeomorphism going from $U=\stackrel{s}{s}\left(\sigma^{n-k-1}, M^{n}\right)$ to

$$
E^{n-k-1} \times\left(\left\{c * L^{k}\right\}-L^{k}\right) \approx E^{n-k-1} \times O \mathscr{C} L^{k}
$$

has the desired properties.

Since $L^{k} \approx S^{k}$, except perhaps for $k=3$ or 4 (refer to Remark 2 ), it is clear that int $\sigma^{n-k-1}$ is locally flat in $M^{n}$, except perhaps for $k=3$ or 4 . We recall, that $L^{4} \times E^{1}$ is a manifold and by assumption $L^{4}$ is homotopy equivalent to $S^{4}$. Hence, as we noted earlier $O \mathscr{C} L^{4} \approx E^{5}$. We can certainly suppose $O \mathscr{C} L^{4} \approx E^{5}$, so that $c$ is carried to the origin of $E^{5}$. Hence, int $\sigma^{n-k-1}$ is locally flat in $M^{n}$, except perhaps for $k=3$.

Clearly, int $\sigma^{n-4}$ is locally flat for $n=4$. We now claim that int $\sigma^{n-4}$ is locally flat for all $n \geqq 5$. That is, we will show that $E^{n-4} \times\left(O \mathscr{C} L^{3}\right) \approx E^{n-4} \times E^{4}$, so that $E^{n-4} \times c$ goes to $E^{n-4} \times \theta_{4}$, where $\theta_{4}$ is the origin of $E^{4}$. This follows for $n=5$, since $E^{1} \times\left(O \mathscr{C} L^{3}\right) \approx$ $E^{1} \times\left(O \mathscr{C} S^{3}\right) \quad($ refer to Remark 1$) \approx E^{1} \times E^{4}$, sending $E^{1} \times c$ to $E^{1} \times \theta_{4}$. Now this holds for any 3 -dimensional link in $M^{n}(n \geqq 6)$ and hence,

$$
\begin{aligned}
E^{n-4} \times\left(O \mathscr{C} L^{3}\right) & =E^{n-5} \times\left(E^{1} \times\left(O \mathscr{C} L^{3}\right)\right) \approx E^{n-5} \times\left(E^{1} \times E^{4}\right) \\
& =E^{n-4} \times E^{4},
\end{aligned}
$$

with $E^{n-4} \times c$ going to $E^{n-5} \times\left(E^{1} \times \theta_{4}\right)=E^{n-4} \times \theta_{4}$.

THEOREM 3. Suppose $M^{n}$ is a simplicial homotopy n-manifold, $N^{k}$ is a simplicial homotopy k-manifold that is a subcomplex of $M^{n}$, and for $n$ or $k=4$ the corresponding complex is also a topological (closed) $n$ or $k$-manifold. If $n-k \geqq 3$, then $N^{k}$ is topologically 
locally flat in $M^{n}$. In particular, if $M^{n} \approx S^{n}$ and $N^{k} \approx S^{k}$, then $\left(M^{n}, N^{k}\right) \approx\left(\Sigma^{n-k} \dot{\Delta}^{k+1}, \dot{\Delta}^{k+1}\right)$.

Proof. The proof is by induction on $k$. The result for $k \leqq 3$ follows from [2] (Theorem 5.4), since $N^{k}$ is $P L$ and each open simplex of $N^{k}$ is locally flat in $M^{n}$ (by Theorem 2).

Now suppose $k=4$ (and hence $n \geqq 7$ ). Let $v$ be a vertex of $N^{4}$ and consider the pair $\left(l k\left(v, M^{n}\right), l k\left(v, N^{4}\right)\right)$. Then $L^{n-1}=l k\left(v, M^{n}\right)$ is a triangulated $(n-1)$-sphere (by Remark 2 ) containing the $P L$ homotopy 3 -spere $L^{3}=l k\left(v, N^{4}\right)$ as a subcomplex. But then, by the first paragraph, $L^{3}$ is locally flat in $L^{n-1} \approx S^{n-1}$. Hence, $O \mathscr{C} L^{n-1} \supset O \mathscr{C} L^{3}$ and $O \mathscr{C} L^{3} \approx E^{4}$ (since $N^{4}$ is a topological 4-manifold) is locally flat in $O \mathscr{C} L^{n-1} \approx E^{n}$, except perhaps for the vertex $v$ of the cone. It follows then that $N^{4} \subset M^{n}$ is locally flat in $M^{n}$ except perhaps for a subset $P^{0}$ of the vertices of $N^{4}$. Since $P^{0}$ is a locally finite polyhedron in int $M, n-4 \geqq 3$, each open simplex of $P^{0}$ is locally flat in int $M^{n}$ and in int $N^{4}, n$-dim $P^{0}>4$, and $N^{k}-P^{0}$ is locally flat in $M^{n}$, it follows by Theorem 5.3 of [2] that $N^{k}$ is locally flat in $M^{n}$ (also see Corollary 7.2 of [3]).

Now suppose $k=5$. We again take a vertex $v \in N^{5}$ and consider the pair $\left(l k\left(v, M^{n}\right),\left(l k\left(v, N^{5}\right)\right)=\left(L^{n-1}, L^{4}\right)\right.$. Then $L^{n-1}$ is a triangulated $(n-1)$-sphere containing the simplicial homotopy 4-manifold $L^{4}$ as a subcomplex. It is not too difficult to see that $L^{4}$ can be considered as a $P L$ 4-manifold modulo a subset $V$ of the vertices of $L^{4}$. Hence, by the above case for $k=4, L^{4}-V$ is locally flat in $L^{n-1}-V$. Therefore, $v * L^{4} \subset v * L^{n-1}$ is locally flat in $v * L^{n-1}$ except perhaps for $v * V$. Thus $N^{5} \subset M^{n}$ is locally flat except perhaps for a subset $P^{1}$ of the 1-skeleton of $N^{5}$. Again it follows by Theorem 5.3 of [2] that $N^{5}$ is locally flat in $M^{n}$ (also see Theorem 7.3 of [3]).

Thus if $N^{k} \subset M^{n}, n-k \geqq 3$ and $k \leqq 5$, then $N^{k}$ is locally flat in $M^{n}$. It follows then that if $M^{n} \approx S^{n}, N^{k} \approx S^{k}, n-k \geqq 3$ and $k \leqq 5$, then $S^{k}$ is flat in $S^{n}$ [15]. We now suppose $k \geqq 6$. Then for every vertex $v \in N^{k},\left(l k\left(v, M^{n}\right), l k\left(v, N^{k}\right)\right)$ is a simplicial homotopy sphere pair. Since $k \geqq 6, l k\left(v, M^{n}\right),\left(l k\left(v, N^{k}\right)\right)$ is also a topological sphere pair, and by induction and [15], $l k\left(v, N^{k}\right)$ is flat in $l k\left(v, M^{n}\right)$. But then,

$$
\operatorname{sit}\left(v, N^{k}\right) \approx O \mathscr{C} l k\left(v, N^{k}\right) \subset O_{\mathscr{C}} l k\left(v, M^{n}\right) \approx \operatorname{st}\left(v, M^{n}\right)
$$

is locally flat and hence, $N^{k}$ is locally flat in $M^{n}$.

Corollary 7. Suppose the answer to the Simplified Double Suspension Problem is always NO. If $M^{n}$ is a triangulated closed topological n-manifold, $N^{k}$ is a closed topological k-manifold that is 
also a subcomplex of $M^{n}$, and $n-k \geqq 3$, then $N^{k}$ is topologically locally flat in $M^{n}$.

This follows immediately from [8] and Theorem 3.

THeOREM 4. (A.) Suppose for some integers $n>k, n-k \neq 2$, there exist a triangulated closed $n$-manifold $M^{n}$ and a closed $k$-manifold $N^{k}$ such that $N^{k}$ is a subcomplex of $M^{n}$, but $N^{k}$ is not locally flat in $M^{n}$ at some point. Then, one of $M^{n}$ or $N^{k}$ is not a simplicial homotopy manifold, and there exists a positive answer to the Simplified Double Suspension Problem.

(B.) Suppose $M^{n}$ is a triangulated closed n-manifold, but is not a simplicial homotopy n-manifold. Then there exists a finite contractible complex $K \subset E^{m} \subset S^{m}$ (for some $m \geqq 4$ ) with $\pi_{1}\left(E^{m}-K\right) \neq 0$ such that $\left(E^{m} / K\right) \times E^{1}$ is locally Euclidean (i.e., a positive answer to the Simplified Double Suspension Problem). Moreover, if $W$ is a regular neighborhood of $K$ in $E^{m}$ and $D$ is a $P L(m-1)$-cell in $B d W$ then

(i ) $\pi_{1}(B d W) \neq 0$ and $\Sigma^{2}(B d W) \approx S^{m+1}$ gives a non-combinatorial triangulation of $S^{m+1}$ and is itself not a simplicial homotopy $(m+1)$ manifold,

(ii) $\Sigma^{2}(B d D)$ is a $P L m$-sphere in the triangulated $(m+1)$-sphere $\Sigma^{2}(B d W)$ such that $\Sigma^{2}(B d D)$ bounds the PL $(m+1)$-cell $\Sigma^{2}(D)$, but $\Sigma^{2}(B d W)-\Sigma^{2}(D)$ is not simply connected, and

(iii) $\Sigma^{2}(B d W)$ is a triangulated $(m+1)$-sphere in the $P L(m+2)$ sphere $\Sigma^{2} S^{n}$ such that one component of $\Sigma^{2} S^{m}-\Sigma^{2}(B d W)$ is homeomorphic to $E^{m+2}$, while the other is not simply connected.

(Thus, if the polyhedral Schoenflies conjecture is false, then there exist counterexamples to the polyhedral Schoenflies conjectures of both type I and type II discussed in [6].)

Proof of (A.). This follows immediately from Theorems 1 and 3 and Corollaries 6 and 7.

Proof of (B.). This follows from the results in [8]. We have already noted the equivalence of the statement that all triangulated manifolds are simplicial homotopy manifolds and the statement that the answer to the Simplified Double Suspension Problem is always NO. The remaining conclusions follow from Theorem II. 2.4, (iii) of [8]. However, since the remaining conclusions are easy to obtain and their proofs are quite illustrative, we will indicate them briefly here.

Hence, suppose $K$ is a finite contractibe complex in $E^{m}$ such that $\pi_{1}\left(E^{m}-K\right) \neq 0$, but $\left(E^{m} / K\right) \times E^{1}$ is locally Euclidean. Since $K$ is contractible, $W$ is a compact contractible $P L m$-manifold with nonempty boundary. By Poincaré duality, $B d W$ has the homology groups of $S^{m-1}$. 
Since $\pi_{1}\left(E^{m}-K\right) \neq 0$, it follows by Van Kampen's theorem that $\pi_{1}(B d W) \neq 0$. Since $W-K \cong B d W \times[0,1$ ) (since $W$ is a regular neighborhood of $K$ and we can also suppose that $K \subset \operatorname{int} W$ ) and $\left(E^{m} / K\right) \times E^{1}$ is locally Euclidean, it follows that $\Sigma(B d W) \times E^{1}$ is locally Euclidean. Hence, $\Sigma^{2}(B d W)$ is a topological $(m+1)$-manifold and by [1], $\Sigma^{2}(B d W) \approx S^{m+1}$ (these ideas are also discussed in greater detail in [9]). Since the link of any vertex in the suspension circle of $\Sigma^{2}(B d W)$ is $\Sigma(B d W)$ and $\pi_{1}(B d W) \neq 0$, it follows that $\Sigma(B d W)$ is not even topologically homeomorphic to $S^{m}$; hence, $\Sigma^{2}(B d W)$ is not a $P L(m+1)$-manifold. Since the link of any 1-simplex in the suspension circle is $B d W$ and $\pi_{1}(B d W) \neq 0, \Sigma^{2}(B d W)$ is not a simplicial homotopy $(m+1)$-manifold.

To see that $\Sigma^{2}(B d W)-\Sigma^{2} D$ is not simply connected, we note that $\Sigma^{2}(B d W)-\Sigma^{2} D \approx(B d W-D) \times E^{2}$ and $\pi_{1}(B d W-D)=$ $\pi_{1}(B d W) \neq 0$. Finally, we consider $K \subset W \subset S^{m}$ and let $V=S^{m}-\operatorname{int} W$. Since $\pi_{1}(V)=\pi_{1}\left(S^{m}-K\right) \neq 0$ and

$$
\Sigma^{2} S^{m}-\Sigma^{2}(B d W) \approx\left(S^{m}-B d W\right) \times E^{2}=\left\{\text { int } W \times E^{2}\right\} \cup\left\{\text { int } V \times E^{2}\right\},
$$

the last conclusion follows. That is, int $V \times E^{2}$ is not simply connected and by [16], int $W \times E^{2} \cong E^{m+2}$.

\section{REFERENCES}

1. M. Brown, Locally flat embeddings of topological manifolds, Ann. of Math., 75 (1962), 331-341.

2. J. L. Bryant, and C. L. Seebeck, Locally nice embeddings of polyhedra, Quart. J. Math., Oxford (2) 19 (1968), 257-274.

3. J. C. Cantrell, and R. C. Lacher, Some conditions for manifolds to be locally flat, (to appear).

4. Marshall M. Cohen, Homeomorphisms between homotopy manifolds and their resolutions, (to appear).

5. E. H. Connell, A topological H-cobordism theorem for $n \geqq 5$, Illinois J. Math., 11 (1967), 300-309.

6. M. L. Curtis, and E. C. Zeeman, On the polyhedral Schoenflies theorem, Proc. Amer. Math. Soc., 11 (1960), 888-889.

7. Robert D. Edwards, and Robin C. Kirby, Deformation of spaces of Imbeddings, (to appear).

8. L. C. Glaser, On suspensions of homology spheres, (to appear).

9. - On the double suspension of certain homotopy 3-spheres, Ann. of Math., 85 (1967), 494-507.

10. - An elementary proof that the double suspension of a homotopy 3-cell is a topological 5-cell, (to appear).

11. Robin, C. Kirby, On the se, of non-locally flat points of a submanifold of codimension one Ann. of Math., 88 (1968), 281-290.

12. Ronald H. Rosen, A note concerning certain subcomplexes of triangulated manifolds, Yokohama Math. J., 16 (1968), 5-7.

13. ——, Concerning suspension spheres Proc. Amer. Math. Soc., 23 (1969), 225231. 
14. L. C. Siebenmann, Are non-trianguable manifolds trianguable?, (to appear).

15. J. Stallings, On topologically unknotted spheres, Ann. of Math., 77 (1963), 490-503.

16. - The piecewise linear structure of Euclidean space, Proc. Cambridge Philos. Soc., 58 (1962), 481-488.

17. E. C. Zeeman, Unknotting combinatorial balls, Ann. of Math., 78 (1963), 501-526.

Received 5, 1970. Work partially supported by the National Science Foundation under NSF Grant GP-11478. The author is also an Alfred P. Sloan Fellow.

The Institute For Advanced Study

AND

UNIVERSITY OF UTAH 



\section{PACIFIC JOURNAL OF MATHEMATICS}

\section{EDITORS}

\author{
H. SAMELSON \\ Stanford University \\ Stanford, California 94305 \\ C. R. HовBY \\ University of Washington \\ Seattle, Washington 98105
}

J. DUGUndis

Department of Mathematics

University of Southern California

Los Angeles, California 90007

RICHARD ARENS

University of California

Los Angeles, California 90024

\section{ASSOCIATE EDITORS}
E. F. BeCKenbaCH
B. H. NeUmanN
F. WOLF
K. YoshidA

\section{SUPPORTING INSTITUTIONS}

\author{
UNIVERSITY OF BRITISH COLUMBIA \\ CALIFORNIA INSTITUTE OF TECHNOLOGY \\ UNIVERSITY OF CALIFORNIA \\ MONTANA STATE UNIVERSITY \\ UNIVERSITY OF NEVADA \\ NEW MEXICO STATE UNIVERSITY \\ OREGON STATE UNIVERSITY \\ UNIVERSITY OF OREGON \\ OSAKA UNIVERSITY \\ UNIVERSITY OF SOUTHERN CALIFORNIA
}

\author{
STANFORD UNIVERSITY \\ UNIVERSITY OF TOKYO \\ UNIVERSITY OF UTAH \\ WASHINGTON STATE UNIVERSITY \\ UNIVERSITY OF WASHINGTON

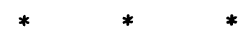 \\ AMERICAN MATHEMATICAL SOCIETY \\ CHEVRON RESEARCH CORPORATION \\ NAVAL WEAPONS CENTER
}

The Supporting Institutions listed above contribute to the cost of publication of this Journal, but they are not owners or publishers and have no responsibility for its content or policies.

Mathematical papers intended for publication in the Pacific Journal of Mathematics should be in typed form or offset-reproduced, (not dittoed), double spaced with large margins. Underline Greek letters in red, German in green, and script in blue. The first paragraph or two must be capable of being used separately as a synopsis of the entire paper. The editorial "we" must not be used in the synopsis, and items of the bibliography should not be cited there unless absolutely necessary, in which case they must be identified by author and Journal, rather than by item number. Manuscripts, in duplicate if possible, may be sent to any one of the four editors. Please classify according to the scheme of Math. Rev. Index to Vol. 39. All other communications to the editors should be addressed to the managing editor, Richard Arens, University of California, Los Angeles, California, 90024.

50 reprints are provided free for each article; additional copies may be obtained at cost in multiples of 50 .

The Pacific Journal of Mathematics is published monthly. Effective with Volume 16 the price per volume (3 numbers) is $\$ 8.00$; single issues, $\$ 3.00$. Special price for current issues to individual faculty members of supporting institutions and to individual members of the American Mathematical Society: $\$ 4.00$ per volume; single issues $\$ 1.50$. Back numbers are available.

Subscriptions, orders for back numbers, and changes of address should be sent to Pacific Journal of Mathematics, 103 Highland Boulevard, Berkeley, California, 94708.

PUBLISHED BY PACIFIC JOURNAL OF MATHEMATICS, A NON-PROFIT CORPORATION

Printed at Kokusai Bunken Insatsusha (International Academic Printing Co., Ltd.), 7-17, Fujimi 2-chome, Chiyoda-ku, Tokyo, Japan. 


\section{Pacific Journal of Mathematics}

Vol. 38, No. $2 \quad$ April, 1971

Richard Davis Anderson and Thomas Ashland Chapman, Extending

homeomorphisms to Hilbert cube manifolds .................. 281

Nguyen Huu Anh, Restriction of the principal series of $\operatorname{SL}(n, \mathbf{C})$ to some

reductive subgroups................................ 295

David W. Boyd, Indices for the Orlicz spaces . . . . . . . . . . . . 315

William Garfield Bridges, The polynomial of a non-regular digraph ...... 325

Billie Chandler Carlson, Robert K. Meany and Stuart Alan Nelson, Mixed

arithmetic and geometric means........................ 343

H. A. Çelik, Commutative associative rings and anti-flexible rings ...... 351

Hsin Chu, On the structure of almost periodic transformation groups ...... 359

David Allyn Drake, The translation groups of n-uniform translation

Hjelmslev planes ................................ 365

Michael Benton Freeman, The polynomial hull of a thin two-manifold . . . . 377

Anthony Alfred Gioia and Donald Goldsmith, Convolutions of arithmetic

functions over cohesive basic sequences .................... 391

Leslie C. Glaser, A proof of the most general polyhedral Schoenflies

conjecture possible ................................

Thomas Lee Hayden and Ted Joe Suffridge, Biholomorphic maps in Hilbert

space have a fixed point ................................ 419

Roger Alan Horn, Schlicht mappings and infinitely divisible kernels ...... 423

Norman Ray Howes, On completeness ...................... 431

Hideo Imai, Sario potentials on Riemannian spaces................ 441

A. A. Iskander, Subalgebra systems of powers of partial universal

algebras.

Barry E. Johnson, Norms of derivations of $\mathscr{L}(\mathrm{X})$.

David Clifford Kay and Eugene W. Womble, Axiomatic convexity theory and relationships between the Carathéodory, Helly, and Radon numbers

Constantine G. Lascarides, A study of certain sequence spaces of Maddox

and a generalization of a theorem of Iyer .............

C. N. Linden, On Blaschke products of restricted growth .

John S. Lowndes, Some triple integral equations ................. 515

Declan McCartan, Bicontinuous preordered topological spaces ......... 523

S. Moedomo and J. Jerry Uhl, Jr., Radon-Nikodým theorems for the Bochner and Pettis integrals ...

Calvin Cooper Moore and Joseph Albert Wolf, Totally real representations

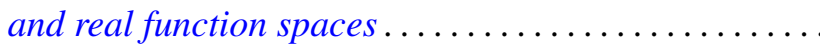

Reese Trego Prosser, A form of the moment problem for Lie groups. ... 